\title{
Competitividad en empresas innovadoras de software y tecnologías informáticas
}

\author{
Competitiveness in innovative software and information technology enterprises
}

Compétitivité dans des entreprises innovatrices de software et de technologies informatiques

\section{Resumen}

Este artículo presenta los resultados del estudio de la “Medición de la competitividad empresarial con indicadores contables”, elaborado por el Grupo de Investigación en Solvencia y Riesgo Financiero de la Universidad del Valle, tomando como universo las empresas que reportaron sus estados financieros a la Superintendencia de Sociedades de Colombia (SSC) del año 2007 al año 2009, específicamente las empresas pertenecientes al sector de software y tecnologías informáticas. Se utilizó la clasificación y datos de la base SIREM de la SSC y se empleó el modelo no paramétrico BCC del Análisis Envolvente de Datos [Data Envelopment Analysis (DEA)]. Los resultados muestran trece empresas técnicamente eficientes en el uso de los recursos tomados como inputs para el año 2007, cuatro empresas eficientes en el año 2008 y diez empresas eficientes para el período 2009; para el análisis de la eficiencia se tomaron como variables de entrada tres y cuatro outputs.

Palabras clave: competitividad, análisis envolvente de datos, eficiencia, indicadores.

\begin{abstract}
This article presents the results of the study "Measuring the Business Competitiveness Financial Indicators" conducted by the Research Group Solvency and Financial Risk at Universidad del Valle, on the universe of companies reporting their financial statements to the Superintendencia de Sociedades de Colombia from 2007 to 2009; specifically, the companies in the software and computer technology field. These results were used for classification and SIREM database of the Superintendency in question and used the nonparametric BCC Data Envelopment Analysis (DEA) model. The results show 13 companies with technically efficient use of resources taken as inputs for 2007, four companies in 2008 were efficient, and 10 effective companies for 2009 . The efficiency analysis considered three variables as input and four outputs.
\end{abstract}

Keywords: competitiveness, data envelopment analysis, efficiency, indicators.

\section{Résumée}

Cet article présente les résultats de l’étude sur la “Mesure de la compétitivité des entreprises avec des indicateurs comptables“, élaborée par le Groupe de Recherche en Solvabilité et Risque Financier de l'Université del Valle. Il considère l'univers des entreprises qui ont reporté ses états financiers à la Superintendencia de Sociedades de Colombia (SSC) [entité étatique qui surveille les sociétés commerciales en Colombie] entre l'année 2007 et l'année 2009, spécifiquement les entreprises appartenant au secteur des technologies informatiques et du software. On a utilisé le classement et les données de la base SIREM de la SSC et on a employé le modèle non paramétrique BCC du Data 
Envelopment Analysis (DEA). Les résultats montrent treize entreprises techniquement efficaces dans l'usage des ressources prises en tant que inputs pour l'année 2007, quatre entreprises efficaces pour l'année 2008 et dix entreprises pour la période 2009. Pour l'analyse de l'efficacité on a pris comme variables d'entrée trois et quatre outputs.

\section{Mots clef: compétitivité, Data Envelopment Analysis (DEA),} efficacité, indicateurs.

\section{Introducción}

Actualmente el comercio mundial se caracteriza por sus altos niveles de competitividad producto de la globalización de los mercados, lo cual ha hecho que los procesos innovadores empresariales en los últimos años hayan tenido gran importancia en los desarrollos dirigidos por las organizaciones. Una empresa que no posea un plan consensuado de innovación de sus actividades operativas y comerciales, en poco tiempo perderá competitividad en el mercado y luego desaparecerá.

Todos los procesos y operaciones que se llevan a cabo en las organizaciones implican transformación, adición de suministros y manipulación de materias primas, para convertirlos en bienes y/o servicios que los consumidores requieren. La transformación implica el uso de -inputsinsumos, mano de obra calificada, materiales, máquinas, energía y otros recursos, y a su vez la generación de salidas -outputs- de productos terminados, servicios, la satisfacción del cliente y otros resultados.

Los gerentes a menudo están interesados en evaluar qué tan eficiente son los diversos procesos y operaciones que se ejecutan con respecto a las variables empleadas (entradas y salidas). Por ejemplo, si se analiza la cadena de suministro entre comprador y vendedor, el comprador puede estar interesado en comparar el desempeño de varios vendedores con respecto al tiempo de respuesta, los costos, la flexibilidad, el servicio al cliente, la calidad y atención personalizada. La eliminación o la mejoría de operaciones y/o procesos ineficientes disminuyen el costo de los insumos (inputs) y aumenta la productividad. La evaluación del desempeño y el análisis comparativo o benchmarking ${ }^{1}$, que de acuerdo con Spendolini (1990), es un proceso de comparación que genera un aprendizaje de las mejores prácticas y que comprende el evaluar, entender, diagnosticar, medir y comparar, que requiere de toda una metodología investigativa, de las operaciones o procesos de las empresas y ayuda a que estas puedan llegar a ser más productivas y eficientes.

A través de la evaluación del desempeño, se puede entre otras cosas:
1. Poner de manifiesto las fortalezas y debilidades de las operaciones, procesos y actividades empresariales.

2. Preparar mejor la empresa para satisfacer las necesidades y requerimientos de los clientes.

3. Identificar oportunidades para mejorar las operaciones y los procesos actuales y crear nuevos productos, servicios y procedimientos.

La evaluación del desempeño es un instrumento importante de mejora continua, una herramienta para mantenerse competitivo y juega un papel importante en un mundo altamente tecnificado, que utiliza intensivamente los equipos de cómputo y las telecomunicaciones, un mundo en donde la competencia es agresiva y crece todos los días. La evaluación del desempeño y el análisis comparativo posibilitan y vigorizan cualquier unidad de negocios a evolucionar y mejorar constantemente con el fin de sobrevivir y prosperar en un ambiente de negocios muy competitivo y globalizado, proceso que ha generado diversas transformaciones a nivel mundial.

La competitividad como un elemento primordial para que las organizaciones sobrevivan en el mundo globalizado, hace fundamental realizar estudios sobre el comportamiento que tienen las empresas en el contexto. De acuerdo con el concepto de eficiencia visto como la obtención de la máxima productividad a los mínimos costos de producción posibles y siendo esta uno de los objetivos principales de toda organización, se quiere enfocar este concepto en la realización del estudio de la competitividad del sector software y tecnologías informáticas.

En el presente estudio se realiza el análisis de eficiencia con un método no paramétrico, el DEA (Análisis Envolvente de Datos) sobre una muestra de empresas catalogadas como innovadoras en el sector software y tecnologías informáticas, con el fin de analizar la eficiencia de este sector entre los años 2007 y 2009 a partir de la información reflejada en los estados financieros de las empresas que reportan sus estados financieros a la Superintendencia de Sociedades de Colombia (SSC); esta metodología se empleó utilizando el paquete matemático Matlab (especializado en el trabajo con matrices de datos), con orientación bajo rendimientos a escala constantes, tomando en cuenta los inputs y output respectivos.

El objetivo del estudio es analizar la eficiencia operativa de las empresas seleccionadas con el propósito de optimizar sus estructura productivas, buscando la máxima producción posible con los recursos que se emplean y servirá para orientar las políticas internas

1. El benchmarking es un anglicismo que, en administración de empresas, puede definirse como un proceso sistemático y continuo para evaluar comparativamente los productos, servicios y procesos de trabajo en organizaciones. Consiste en tomar "comparadores" o benchmarks a aquellos productos, servicios y procesos de trabajo que pertenezcan a organizaciones que evidencien las mejores prácticas sobre el área de interés, con el propósito de transferir el conocimiento de las mejores prácticas y su aplicación. 
y los procesos de toma de decisiones por medio del benchmarking, con el fin de implementar un adecuado control de gestión y mejorar la competitividad en el mercado local y mundial.

A través del estudio se quiere brindar información a las empresas del sector software y tecnologías informáticas acerca de su posicionamiento frente al comportamiento de eficiencia ideal que debe manejar este sector, permitiendo identificar las posibles causas de eficiencia o ineficiencia que pueden presentar las empresas siendo una herramienta útil para el nivel estratégico o alta gerencia debido a que proporciona lineamentos necesarios para la toma de decisiones acerca del diseño de políticas que contribuyan a mejorar su desempeño y a la satisfacción de las necesidades de los agentes internos y externos de la empresa para el logro de metas compartidas de progreso y modernidad, es decir, la capacidad de crecer con calidad tanto a nivel corporativo como regional.

La muestra empleada utiliza datos de empresas clasificadas como innovadoras en software y son aquellas cuyas características generadas en el inicio de un proyecto de innovación cubre una variedad de aspectos, algunos tienen relación con los cambios que se requieren en la administración para el desarrollo, implementación y operación del nuevo sistema (Richard, 2002), además de asuntos como comunicación, coordinación, etc. Las principales características para hacer integración son:

* Una redefinición de la arquitectura interna de la empresa alrededor de nuevas competencias requeridas por el proyecto. De ese modo dos características de innovación son: a) Nuevos procedimientos y el aumento de los cruces funcionales y la integración y b) Un inicial aumento de las prácticas establecidas.

* Una redefinición del valor de la cadena orientado alrededor del nuevo rol de los proveedores de tecnología, desarrolladores de software y compañías de internet. Esto representa un cambio en las tradicionales operaciones de la industria de telecomunicaciones. La innovación ahora requiere de la administración de una red de proveedores y de recursos externos. De este modo una característica de la innovación obtenida significa una red de proveedores interdependientes.

* Una orientación hacia el mercado sugiere una cultura centrada en la innovación en la firma, que permite establecer conductas necesariamente más eficientes y eficaces para la creación de valor superior para los compradores y así, de esta manera, mantener un desempeño superior sustentable para el negocio.

Las características de los servicios y actividades de los negocios innovadores son: la información que se basa en la natural asociación con las tecnologías de la informática y la computación, la flexibilidad de servicios debido a la interacción entre los proveedores y los usuarios del servicio y el papel fundamental del recurso humano y la estimulación constante paraquela firma de respuestas innovadoras (Consoli, 2010).

El desarrollo de nuevos productos es crucial para la renovación, supervivencia y éxito de las organizaciones. Para continuar con el éxito en el desarrollo de nuevos productos, las necesidades de mercadeo tienen una significativa influencia. Sin embargo, el mercadeo con frecuencia finaliza con un papel secundario para la ingeniería. Una gran coordinación entre las áreas funcionales es esencial para el exitoso desarrollo de nuevos productos (Sarin y Mahajan, 2001).

Muller et al. (2009) encontraron que el sector de alta tecnología ahora presenta un cambio significativo en la manera cómo las firmas ganan acceso al mercado teniendo como clave la experticia. El crecimiento y la diversidad de actividades de servicios en los negocio refleja un modo de operación moderno donde la alta experticia juega un papel clave para las pequeñas compañías, con el fin de contrarrestar cualquier esfuerzo de las grandes organizaciones para dominar algunos sectores del mercado.

Se puede luego decir que la evaluación del desempeño es un instrumento importante de mejora continua, una herramienta para mantenerse competitivo y juega un papel importante en un mundo altamente tecnificado, que utiliza intensivamente los equipos de cómputo y las telecomunicaciones, un mundo en donde la competencia es agresiva y crece todos los días.

La competitividad de acuerdo con Álvarez (2002) es la capacidad de toda organización de mantener sistemáticamente ventajas comparativas que le permitan alcanzar, sostener y mejorar una determinada posición en el entorno socioeconómico, la cual implica la determinación de los componentes o factores que la generan y el grado de impacto de los mismos; para poder medir la competitividad que tiene la organización en el mercado es necesario evaluar tanto la gestión interna a través de indicadores financieros tales como operacionales o de actividad, de liquidez, de endeudamiento, de rentabilidad, como la gestión externa y el desempeño respecto a otras empresas.

A través de los años el estudio de la competitividad ha dado forma al desarrollo de metodologías que miden diversos aspectos de la misma; una de las herramientas usadas es la técnica de frontera eficiente, la cual se utiliza para obtener información sobre el desempeño y analizar el nivel de competitividad que tiene la organización en el mercado, basándose en la eficiencia como el máximo aprovechamiento de los recursos para obtener mayor productividad.

La evaluación del desempeño y el análisis comparativo posibilitan y vigorizan cualquier unidad de negocios a evolucionar y mejorar constantemente con el fin de sobrevivir y prosperar en un ambiente de negocios muy competitivo y globalizado, proceso este que ha generado diversas transformaciones a nivel mundial y del cual no ha sido ajeno Colombia. 


\section{Metodología}

La elaboración de un análisis de competitividad es un proceso que consiste en relacionar a la empresa con su entorno. Este tipo de análisis ayuda a identificar las fortalezas y debilidades de la empresa, así como las oportunidades y amenazas que le afectan dentro de su mercado objetivo (González, 2010).

El término de eficiencia técnica fue introducido en la literatura económica por Koopmans (1951), afirmando que una combinación factible de inputs y outputs es técnicamente eficiente, si es tecnológicamente imposible aumentar algún output y/o reducir algún input sin reducir simultáneamente al menos otro output $\mathrm{y} / \mathrm{o}$ aumentar al menos otro input.

Inspirado en los trabajos de Koopmans (1951), Farrell (1957) añadió a la eficiencia técnica un nuevo concepto, el de eficiencia asignativa, que él llamó eficiencia en precios. Para ello supuso que la empresa persigue un objetivo que consiste en la minimización de los costes. La eficiencia asignativa consiste en elegir, de entre las combinaciones de inputs y outputs técnicamente eficientes, aquella que resulta más barata según los precios de los inputs.

La gran contribución de Farrell (1957), que le convierte en el autor más influyente en el estudio de eficiencia productiva, consiste en proponer una medición empírica de la eficiencia técnica, que consistió en seleccionar de una muestra de empresas aquellas que tuvieran la mejor eficiencia y construir con éstas una frontera eficiente. Posteriormente, Farrell propuso comparar los resultados obtenidos para cada empresa con la frontera eficiente: entonces, aquellos casos que representen desviaciones de ese "estado ideal" son caracterizados como ineficientes. Por esta razón, se afirma que la medición de la eficiencia de una empresa tiene un carácter relativo, porque debe ser comparado con una medida estándar incluida en la muestra objeto de estudio.

Para realizar este análisis de competitividad en el sector de software y tecnologías informáticas es necesario utilizar un método de estimación para construir la frontera eficiente, que puede ser paramétrico y noparamétrico; para esta investigación será un método no paramétrico (DEA), lo que significa que no se va a tener indicadores de ajuste o contraste como suele utilizarse en los modelos econométricosLa fuente de información a la que se acudió para la obtención de los datos es la base de datos SIREM de la SSC, que es de tipo secundaria y es una base de datos pública, donde aparecen consignadas las cifras de desempeño financiero en los estados financieros respectivos de cada organización que reporta información a esta entidad, además presenta otros indicadores de cada una de la $\mathrm{DMU}^{2}$ analizadas en el período 2007 a 2009.

\subsection{Definicion y tamaño de la muestra}

Para la selección de las DMU, se empleó inicialmente como técnica de recolección, la delimitación por criterios de inclusión; el primero es empresas que reportan sus estados financieros a la SSC; el segundo criterio es tener completos los estados financieros en los períodos analizados. De acuerdo con la información reportada en el SIREM, la población total objeto de estudio son 70 empresas, correspondientes al período 2007 y 2009. Para determinar el tamaño de la muestra en esta investigación se utilizó el muestreo aleatorio simple, que asegura una muestra representativa y produce una estimación de la cantidad de la población.

Inicialmente se calculó el tamaño de la muestra teniendo en cuenta un nivel de confianza del $90 \%$ y un error de estimación del 10\%. El tamaño de la muestra se calculó bajo el criterio de máxima varianza para la estimación de una proporción. Para este concepto se aplica la siguiente expresión:

$\mathrm{n}=\frac{\mathrm{Z}_{\mathrm{a} / 2}^{2} \mathrm{PQ}}{\mathrm{E}^{2}}$

En donde:

$\mathrm{n}$ = Tamaño de la muestra máximo para la estimación de una proporción.

$Z$ = Percentil de la distribución normal equivalente a un nivel de confianza del $90 \%$

$P Q=E s$ la varianza de la proporción, donde: $P$ representa la proporción de empresas que cumplen con las características de evaluación: $Q=1-P$. El valor de $P Q$ es máximo cuando $P=0.50$ y $Q=1-P=0.50$, por tanto $\mathrm{PQ}=0.25$

$E=$ Error de muestreo, es la diferencia entre el valor del parámetro que se desconoce y el valor de estimador que se está encontrando, para este caso este valor es de o.10.

Después de utilizar la fórmula anterior, se aplicó el concepto de tamaño de muestra corregido por población finita, aplicando en la siguiente expresión:

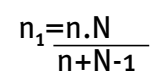

En donde:

$\mathrm{n}_{1}=$ Tamaño de la muestra corregido por población finita.

$\mathrm{n}$ = Tamaño de la muestra máximo para la estimación de una proporción

$\mathrm{N}=$ Población total

Después de haber aplicado el concepto de tamaño de muestra máximo para la estimación de una proporción y

2. Las unidades de decisión llamadas DMU (Decisión Making Units), es el término que emplearon Charnes, Cooper y Rhodes (1978) y representan cualquier organización, agencia, programa o departamento funcional. Las DMU son un grupo de unidades organizacionales donde la presencia de múltiples entradas y salidas (inputs y outputs) hacen difícil la comparación de su desempeño. 
el concepto de tamaño de la muestra corregido por población finita, se podrá sustentar por qué haber escogido la cantidad de 40 empresas como tamaño de la muestra.

$\mathrm{n}=\mathrm{Z}^{2} \mathrm{PQN}$

$(\mathrm{N}-1) \mathrm{E}^{2}+\mathrm{Z}^{2} \mathrm{P} Q$

En donde:

$\mathrm{n}=$ Tamaño de muestra

$Z$ = Valor $Z$ de la curva normal (1.96)

$\mathrm{P}=$ Probabilidad de éxito (0.50)

$\mathrm{Q}=$ Probabilidad de fracaso $(0.50)$

$\mathrm{N}=$ Población (70)

$\mathrm{E}=$ Error muestral (0.10)

$\mathrm{n}=(1.96) 2(0.50)(0.50)(70) .=40$

$(70-1)(0.10) 2+(1.96) 2(0.5)(0.5)$

El tamaño de la muestra corresponde a 40 empresas dedicadas a la industria del software y tecnologías informáticas.

\subsection{Definicion de inputs y outputs}

Teniendo en cuenta que para el análisis de desempeño desde el punto de vista contable financiero, se entiende el sistema de inputs y outputs como una extensión del modelo contable, donde el total de los montos en cuentas del crédito son iguales al total de los montos en las cuentas débito, para el estudio y la construcción de una tabla de inputs y outputs, los débitos y los créditos corresponderán a los inputs y los outputs de cada una de las entidades observadas; por ejemplo, una operación de venta en una entidad implica que la salida de mercancía y/o servicios sea tenida en cuenta como outputs y simultáneamente como un input debido a los ingresos monetarios que se generan, así, el total de los input y outputs se verán reflejados en el balance general. Los input y output para este trabajo corresponden a los utilizados por Quintero (2008) en su investigación.

\begin{tabular}{|c|c|c|c|}
\hline $0 / 1$ & Descripción & Definición & Cálculo \\
\hline Output1 & Ingresos operacionales & $\begin{array}{l}\text { Valores recibidos y/o causados, resultado de las actividades } \\
\text { desarrolladas en cumplimiento del objeto social . }\end{array}$ & \\
\hline Output2 & Utilidad operacional & $\begin{array}{l}\text { Se refiere exclusivamente a ingresos y gastos operacionales, } \\
\text { dejando de lado los gastos e ingresos no operacionales. }\end{array}$ & $\begin{array}{l}\text { Ventas } \\
\text { (-) Devoluciones en ventas } \\
\text { (-) Costo de venta } \\
\text { (-) Gastos operacionales } \\
\text { = Utilidad operacional }\end{array}$ \\
\hline Output3 & Utilidad neta & $\begin{array}{l}\text { Es la resultante después de restar y sumar de la utilidad } \\
\text { operacional, los gastos e Ingresos no operacionales } \\
\text { respectivamente, los impuestos y la reserva legal. Es la utilidad que } \\
\text { efectivamente se distribuye a los socios. La utilidad neta es el } \\
\text { rendimiento o la rentabilidad efectiva que los socios o dueños de } \\
\text { una empresa obtienen por sus aportes. Es lo que los activos de una } \\
\text { empresa generaron en el período respectivo. }\end{array}$ & $\begin{array}{l}\text { Ventas } \\
\text { (-) Devoluciones en ventas } \\
=\text { Ventas netas } \\
\text { (-) Costo de venta } \\
=\text { Utilidad bruta } \\
\text { (-) Gastos operacionales } \\
=\text { Utilidad operacional } \\
\text { (+) Ingresos no operacionales } \\
\text { (-) Gastos no operacionales } \\
=\text { Utilidad antes de impuestos y } \\
\text { reservas } \\
\text { (-) Impuesto renta } \\
\text { (-) Reserva legal } \\
=\text { Utilidad neta }\end{array}$ \\
\hline Input1 & Activo corriente & $\begin{array}{l}\text { Activos susceptibles de convertirse en efectivo en un período inferior } \\
\text { a un año. }\end{array}$ & \\
\hline Input2 & $\begin{array}{l}\text { Propiedad, planta y } \\
\text { equipo }\end{array}$ & $\begin{array}{l}\text { Bienes que posee la empresa para emplearlos en el desarrollo del } \\
\text { giro normal de sus negocios. }\end{array}$ & \\
\hline Input3 & Pasivo no corriente & $\begin{array}{l}\text { Compromisos cuyo vencimiento es mayor a un año. Ejemplo de } \\
\text { estos activos las Obligaciones bancarias LP, Obligaciones por } \\
\text { emisión de bonos LP, etc. }\end{array}$ & \\
\hline
\end{tabular}


La Tabla 1 muestra los input y outputs utilizados en el estudio y aportados por cada empresa y la forma como se obtienen.

\subsection{Selección del modelo DEA}

El análisis envolvente de datos (DEA) es una poderosa técnica no paramétrica de optimización mediante la "función frontera", que utiliza la programación lineal para la construcción de una superficie envolvente, frontera eficiente o función de producción empírica eficiente, que permite medir la eficiencia relativa de las distintas organizaciones a partir de los datos disponibles como las variables de entrada y salida (inputs y outputs) del conjunto de entidades o empresas objeto de estudio. En otras palabras, esta herramienta permite construir la frontera eficiente a partir de las DMU que presenten las mejores prácticas, es decir, aquellas que obtienen el nivel máximo de outputs con los inputs que utilizan, de forma que se pueda medir la ineficiencia del resto de las unidades como distancia a la frontera.

Para medir la eficiencia, el DEA se apoya en la observación de datos, la aplicación de técnicas de programación matemática, el uso de una frontera de referencia (frontera eficiente) y un concepto de distancia de cada DMU a dicha frontera que define el nivel de eficiencia para cada una de las observaciones.

Esta herramienta permite analizar la relación de la producción con la eficiencia productiva sin aplicar una distribución explícita de los términos estadísticos de ineficiencia o una forma funcional de relación entre múltiples inputs y outputs expresados en diferentes unidades.

Así, se compararan el mejor desempeño, producto de la combinación lineal de todas las unidades de la muestra con el rendimiento de los datos observados en las unidades de referencia (DMU). En esta comparación, se asignará una puntuación de eficiencia según la distancia desde la DMU a la frontera de producción estimada, indicando el uso eficiente de las unidades, todo este proceso en aras de la gestión significará un benchmarking para cada una de las unidades estudiadas.

Un requisito principal que exige este modelo es que todas las DMU sean lo más homogéneas posible, es decir, que consuman los mismos tipos de entradas o inputs y produzcan las mismas clases de salidas u outputs, por lo que previamente deben detectarse aquellas unidades que tengan un comportamiento atípico (outliers) para eliminarlas del análisis, porque éstas darían lugar a distorsiones del análisis propuesto y por ende a apreciaciones no concordantes con la realidad.

Una de las ventajas que aporta la medida de la eficiencia a través del modelo DEA, es la posibilidad que ofrece a los gestores de las empresas del sector software y tecnologías informáticas para conocer su posición relativa con respecto al resto de entidades con las que se compara, además de saber las causas del origen de la ineficiencia, en el caso de que exista.

Por otra parte, el modelo DEA orienta la política de los gestores a fin de controlar la desviación producida entre los valores observados, o recogidos empíricamente y los valores obtenidos del modelo a través de la elección de estándares de la mejor práctica implantada. Esto sirve para que las entidades ineficientes puedan tomar las decisiones oportunas de acometer alguna mejora a fin de alcanzar la frontera eficiente.

El modelo BCC (Banker, Charnes y Cooper, 1984), es un modelo orientado a inputs donde son minimizados y los outputs mantenidos en sus niveles corrientes. Es un modelo que incluye procedimientos de programación lineal que permite analizar la frontera de eficiencia a través de varios inputs y outputs cuando todos los DMU cambian simultáneamente.

Este modelo se le puede llamar medida radial de eficiencia porque ajusta todos los inputs y outputs de una DMU en proporciones (pesos), estimando la puntuación de eficiencia técnica pura (ETP). El modelo cumple con los siguientes requerimientos:

$$
\begin{aligned}
& \theta^{*}=\theta \\
& \text { Sujeto a: } \\
& \sum_{j=1}^{n} \lambda_{j} X_{i j} \leq \theta X_{i o} \quad i=1,2 \ldots m \\
& \sum_{j=1}^{n} \lambda_{j} X_{r j} \geq Y_{r o} \quad r=1,2 \ldots s \\
& \sum_{j=1}^{n} \lambda_{j}=1 \\
& \lambda_{j} \geq 0 \quad j=1,2 \ldots n
\end{aligned}
$$

Donde: $\lambda \mathrm{j}$ es el peso de cada input/output y

tomará valores no negativos

$X$ es cada uno de los inputs

Y es cada uno de los outputs

Este será el modelo utilizado para encontrar la eficiencia de cada unidad productiva

\section{Resultados}

Una vez obtenidos los inputs y outputs utilizados por cada DMU se usó el software Matllab para el cálculo de las unidades eficientes, que son las que conformarán la frontera eficiente para cada período analizado. El modelo empleado fue el BCC y en la Tabla 2 se muestran la unidades técnicamente eficientes y 
las que no cumplen este propósito; en este sentido podemos hablar de las unidades que son competitivas en el sector productivo que se encuentran y las que aún no logran estar a la vanguardia en cuanto a la generación de los outputs evaluados (ingresos, utilidad operacional, utilidad neta).

\begin{tabular}{|c|c|c|c|c|c|}
\hline \multicolumn{2}{|c|}{2007} & \multicolumn{2}{|c|}{2008} & \multicolumn{2}{|c|}{2009} \\
\hline DMU & Eficiencia & DMU & Eficiencia & DMU & Eficiencia \\
\hline $\mathrm{DMU}_{1}$ & 1,0000 & $\mathrm{DMU}_{1}$ & 0,9620 & $\mathrm{DMU}_{1}$ & 1,0000 \\
\hline $\mathrm{DMU}_{2}$ & 0,5598 & $\mathrm{DMU}_{2}$ & 0,6286 & $\mathrm{DMU}_{2}$ & 0,9767 \\
\hline $\mathrm{DMU}_{3}$ & 1,0000 & $\mathrm{DMU}_{3}$ & 0,7911 & $\mathrm{DMU}_{3}$ & 1,0000 \\
\hline $\mathrm{DMU}_{4}$ & 0,7325 & $\mathrm{DMU}_{4}$ & 0,7276 & $\mathrm{DMU}_{4}$ & 0,9358 \\
\hline $\mathrm{DMU}_{5}$ & 0,7890 & DMU6 & 0,8304 & $\mathrm{DMU}_{5}$ & 0,7437 \\
\hline DMU6 & 0,0619 & DMU9 & 1,0000 & DMU6 & 0,6803 \\
\hline $\mathrm{DMU}_{7}$ & 0,7790 & DMU10 & 0,6337 & $\mathrm{DMU}_{7}$ & 0,0000 \\
\hline DMU8 & 0,7654 & DMU11 & 1,0000 & DMU8 & 1,0000 \\
\hline DMU9 & 1,0000 & $\mathrm{DMU}_{12}$ & 0,8571 & DMU9 & 0,7494 \\
\hline $\mathrm{DMU}_{10}$ & 0,8722 & DMU14 & 0,6150 & DMU10 & 1,0000 \\
\hline $\mathrm{DMU}_{11}$ & 0,8480 & $\mathrm{DMU}_{16}$ & 0,4323 & DMU11 & 0,7837 \\
\hline $\mathrm{DMU}_{12}$ & 0,2154 & $\mathrm{DMU}_{17}$ & 0,3557 & $\mathrm{DMU}_{12}$ & 0,5561 \\
\hline $\mathrm{DMU}_{13}$ & 1,0000 & DMU18 & 0,6578 & $\mathrm{DMU}_{13}$ & 0,6462 \\
\hline DMU14 & 1,0000 & DMU19 $_{19}$ & 0,7795 & $\mathrm{DMU}_{14}$ & 0,3789 \\
\hline $\mathrm{DMU}_{15}$ & 1,0000 & $\mathrm{DMU}_{20}$ & 0,8757 & $\mathrm{DMU}_{15}$ & 0,6524 \\
\hline $\mathrm{DMU}_{16}$ & 1,0000 & DMU21 & 0,9129 & $\mathrm{DMU}_{16}$ & 1,0000 \\
\hline $\mathrm{DMU}_{17}$ & 0,7916 & $\mathrm{DMU}_{22}$ & 0,4746 & $\mathrm{DMU}_{17}$ & 0,7607 \\
\hline $\mathrm{DMU}_{18}$ & 1,0000 & $\mathrm{DMU}_{23}$ & 0,0000 & $\mathrm{DMU}_{18}$ & 0,7698 \\
\hline $\mathrm{DMU}_{19}$ & 0,5333 & $\mathrm{DMU}_{24}$ & 0,6113 & DMU19 & 0,3914 \\
\hline DMU20 & 1,0000 & $\mathrm{DMU}_{25}$ & 0,1775 & DMU20 & 1,0000 \\
\hline $\mathrm{DMU}_{21}$ & 0,4482 & $\mathrm{DMU}_{26}$ & 1,0000 & DMU21 & 1,0000 \\
\hline DMU22 & 1,0000 & $\mathrm{DMU}_{27}$ & 0,3368 & $\mathrm{DMU}_{22}$ & 0,5907 \\
\hline $\mathrm{DMU}_{23}$ & 0,8754 & $\mathrm{DMU}_{28}$ & 0,0000 & $\mathrm{DMU}_{23}$ & 0,0000 \\
\hline DMU24 & 0,3743 & $\mathrm{DMU}_{31}$ & 0,6775 & DMU24 & 0,8441 \\
\hline $\mathrm{DMU}_{25}$ & 1,0000 & $\mathrm{DMU}_{32}$ & 0,1071 & $\mathrm{DMU}_{25}$ & 1,0000 \\
\hline DMU26 & 1,0000 & $\mathrm{DMU}_{33}$ & 0,9662 & $\mathrm{DMU}_{26}$ & 0,5811 \\
\hline $\mathrm{DMU}_{27}$ & 1,0000 & $\mathrm{DMU}_{34}$ & 0,8337 & $\mathrm{DMU}_{32}$ & 0,0605 \\
\hline $\mathrm{DMU}_{28}$ & 0,5657 & $\mathrm{DMU}_{35}$ & 1,0000 & $\mathrm{DMU}_{33}$ & 1,0000 \\
\hline DMU29 & 0,8515 & $\mathrm{DMU}_{36}$ & 0,4922 & $\mathrm{DMU}_{34}$ & 0,7838 \\
\hline $\mathrm{DMU}_{30}$ & 0,9828 & $\mathrm{DMU}_{37}$ & 0,4763 & $\mathrm{DMU}_{36}$ & 0,8935 \\
\hline \multirow[t]{3}{*}{$\mathrm{DMU}_{31}$} & 0,0000 & $\mathrm{DMU}_{3} 8$ & 0,5191 & $\mathrm{DMU}_{37}$ & 0,7828 \\
\hline & & $\mathrm{DMU}_{39}$ & 0,3935 & $\mathrm{DMU}_{3} 8$ & 0,9030 \\
\hline & & & & $\mathrm{DMU}_{39}$ & 1,0000 \\
\hline
\end{tabular}

En la Tabla 2 se muestra el nivel de eficiencia obtenido por cada una de las DMU analizadas en cada año. Mediante la aplicación del modelo BCC, se obtuvo que e $41.9 \%$ de las empresas innovadoras en software son eficientes (Eficiencia=1) en el año 2007 , estas determinan la frontera eficiente a la cual deberían de aproximarse aquellas unidades no eficientes (eficiencia (1). En cuanto al consumo de los inputs y producción de outputs para el año $2008,12.5 \%$ de las DMU resultaron eficientes; para el año 2009 30.3\% de las DMU analizadas son eficientes; algunas cifras adicionales se presentan en la Tabla 3.

\begin{tabular}{|c|r|r|r|}
\hline Tabla 3. Estadígrafos eficiencia anual \\
\hline & $\mathbf{2 0 0 7}$ & $\mathbf{2 0 0 8}$ & $\mathbf{2 0 0 9}$ \\
\hline & Efficiency & Efficiency & Efficiency \\
\hline Count & 31 & 32 & 33 \\
\hline Mean & 0,775676 & 0,628911 & 0,741353 \\
\hline Sample variance & 0,085749 & 0,087525 & 0,085892 \\
\hline Sample standard deviation & 0,292830 & 0,295847 & 0,293073 \\
\hline Minimum & 0 & 0 & 0 \\
\hline Maximum & 1 & 1 & 1 \\
\hline Range & 1 & 1 & 1 \\
\hline Fuente: Mosquera, H.A. y Millán, J.C. (2013) basado en Megastat.
\end{tabular}

Como se puede observar en la Tabla 3, a pesar de que la muestra fue de 40 empresas al analizar los datos con el aplicativo Megastat para Excel, se presentaron en cada período valores extremos (outliers) esto redujo los datos a 31 en 2007, 32 en 2008 y 33 en 2009. Comparando los tres años se nota que el año 2007 es el menos heterogéneo porque presenta un coeficiente de variación de $38 \%$ obtenido como el cociente entre la desviación estándar muestral y la media de los datos analizados; sigue el año 2009 con $40 \%$ y el más heterogéneo es el año 2008 con $47 \%$.

Para el año 2007 resultaron 13 empresas eficientes, 4 en el 2008 y 10 para el año 2009, este grupo de DMU conforma la frontera eficiente (técnicamente competitivas).

\section{Conclusiones}

La metodología DEA ha permitido realizar un análisis de competitividad basándose en las organizaciones que mejor operan o administran los recursos y obtienen buenos resultados en el grupo de empresas seleccionadas, lo que propicia sugerir la mejora en el empleo de los inputs utilizados (activo corriente, propiedad planta y equipo, pasivo, no corriente, patrimonio) por las empresas innovadoras en software y tecnologías de información identificadas como ineficientes, con el objeto de servir de guía para su gestión futura.

Se concluye que las DMU ineficientes pueden mejorar su eficiencia adoptando medidas estratégicas adecuadas para obtener la mejor combinación de recursos. La transmisión de las características de las empresas eficientes posibilita el mejoramiento de la competitividad de las empresas en el sector evaluado.

La frontera eficiente o función de producción empírica permite medir la eficiencia relativa de las distintas organizaciones a partir de los datos disponibles (inputs y outputs) del conjunto de entidades objeto de estudio. Es una herramienta que permite construir la frontera eficiente a partir de las DMU que presenten las mejores prácticas, es decir, aquellas que obtienen el nivel máximo de outputs con los inputs que utilizan, de forma que se pueda medir la ineficiencia del resto de las unidades como distancia a la frontera. 
Los outputs que presentaron ineficiencia entre los años 2007 y 2009) son el output 2 "utilidad operacional" y el output 3 "utilidad neta". Se recomienda a las empresas que quedaron catalogadas como ineficientes concentrar sus esfuerzos en el mejoramiento o alcance de la eficiencia, esto significará de un lado, adecuar la estructura operativa de la organización con el fin de permitir que la financiación vía recursos de terceros (pasivo financiero) no disminuya significativamente la utilidad neta y por tanto el beneficio de los propietarios.

\section{Referencias}

Adams, A.A. \& Stewart, I.G. (1956). Input-Output Analysis: An Application. Recuperado 04/05/2012 de: http://www.jstor.org/ stable/2227861

Acevedo Villalobos, M.C. y Ramírez, Vallejo, J. (2005). Diferencias regionales en la eficiencia técnica del sector confecciones en Colombia: un análisis de fronteras estocásticas. Innovar. Revista de Ciencias Administrativas y Sociales, 15 (26) (pp. 90-105).

Álvarez-Pinilla, A. (2002). La medición de la eficiencia y la productiva. Madrid, España: Editorial Pirámide.

Asociación Nacional de Centros Europeos de Innovación Españoles (ANCES). (2003). La creación de empresas de base tecnológica: una experiencia práctica. Madrid, España: ANCES.

Banker, R.D., Charnes, A. \& Cooper, W.W. (1984). Some models for estimating technical and scale inefficiencies in DEA. Management Science, 30 (9) (pp. 1078-1092).

Bendapudi, N. \& Berry, L.L. (1997). Customers' Motivations for maintaining relationship with service providers. Journal of Retailing, 73 (1) (pp. 15-37).

Berry, L.L. y Parasuraman, A. (1994). Marketing en las empresas de servicio: compita mediante la calidad. Jorge Cardenas Nannetti (Trad.). Bogotá, Colombia: Editorial Norma.

Bitner, M.J. (1990). Evaluating service encounters: the effects of physical surrounding and employee responses. Journal of Marketing, 54 (pp. 69-82).

Bolton, R.N. \& Drew, J.H. (1991). A multistage model of customers' assessments of service quality and value. The Journal of Consumer Research, 17 (4) (pp. 375-384).

Coll Serrano, V. y Blasco, O.M. (2006). Evaluación de la eficiencia mediante el análisis envolvente de datos. Recuperado 02/03/2012 de:http://www.eumed.net/libros/2006c/197/ index.htm

Consoli, D. \& Elche-Hortelano, D. (2010). Variety in the knowledge base of Knowledge Intensive Business Services. Research Policy, 39 (10) (pp. 1303-1310).

Eccles, R.G. (2004). Manifiesto sobre la medida del rendimiento". Cómo medir el rendimiento en la empresa. Barcelona, España: Harvard Business Review, Ediciones Deusto S.A.

Caves, D.W., Christensen, L.R. \& Erwin Diewert, W. (1982). The Economic Theory of Index Numbers and the Measurement of Input, Output, and Productivity. Econometrica, 50 (6) (pp. 1393-1414). Recuperado 15/03/2012 de: http://www.jstor.org/stable/1913388
Galagedera, D.U.A. \& Silvapulle, P. (2003). Experimental evidence on robustness of data envelopment analysis. The Journal of the Operational Research Society, 54 (6) (pp. 654-660). Recuperado 16/03/2012 de: http://www.jstor.org/stable/4101757

González, R.M. (2010). Marketing en el Siglo XXI. Recuperado 1/4/2011 de: http://www.marketing-xxi.com/analisis-competitivo-17.htm

Gronroos, C. (2000). Relationship marketing: interaction, dialoge and value. Revista Europea de Dirección y Economía de la Empresa, 9 (3) (pp. 13-24).

Holsapplea, C.W. \& Joshib, K.D. (2000). An investigation of factors that influence the management of knowledge in organizations. Journal of Strategic Information Systems, 9 (pp. 235-261).

Richards, A.B. (1960). Input-output accounting for business. The Accounting Review, 35 (3) (pp. 429-436). Recuperado 20/03/2012 de: http://www.jstor.org/stable/242577

Jaw, C., Lo, J.Y. \& Lin, YH. (2010). The determinants of new service development: Service characteristics, market orientation, and actualizing innovation effort. Technovation, 30, (4) (pp. 265-277).

Könnölä, T., Brummer, V. \& Salo, A. (2007). Diversity in foresight: Insights from the fostering of innovation ideas. Technological Forecasting \& Social Change, 74 (pp. 608-626).

Farrell, M.J. (1957). The measurement of productive efficiency. Journal of the Royal Statistical Society, Series A, 120 (3), (pp. 253-290). Recuperado 20/03/2012 de: http://www.jstor.org/ stable/2343100

Muller, E. \& Doloreux, D. (2009). What we should know about knowledge-intensive business services. Technology in Society, 31. (pp 64 - 72).

Pan Feng, C. \& Chen Chi, S. (2004). Enhancing competitive adventage of hospitals through linguistics evaluation on customer perceived value. The Jounal of American Academy of Business, (9) (pp. 481-485).

Quintero-Otero, J. (2008). Competitividad en la industria manufacturera de Cartagena: Un análisis de eficiencia técnica en el periodo 2001-2004. Cartagena, Colombia: Observatorio del Caribe Colombiano.

Sarin, S. \& Mahajan, V. (2001). The effect of reward structures on the perfomance of cross - functional product development teams. Journal of Marketing, 65 (pp. 35-53).

Senge, P. (2005). La Quinta Disciplina: el arte y la práctica de las organizaciones abiertas al aprendizaje. Buenos Aires, Argentina: Ediciones Gránica S.A.

Spendolini, M. (1990). Benchmarking. Bogotá, Colombia: Editorial Norma S.A.

Rodríguez-Lozano, G.I. (2003). Medición de la eficiencia relativa en tres subsectores de la economía colombiana desde 1993 a 1999 utilizando Data Envelopment Analysis (D.E.A.).Innovar. Revista de Ciencias Administrativas y Sociales, 13 (21) (pp. 145-181).

Truffer B., Voß, J.P. \& Konrad, K. (2008). Mapping expectations for system transformations lessons from sustainability foresight in german utility sectors. Technological Forecasting \& Social Change, 75 (pp. 1360-1372).

Cuadernos de Administración / Facultad de Ciencias de la Administración / Universidad del Valle

Periodicidad: semestral / ISSN impreso $N^{\circ}$ 0120-4645-ISSN electrónico N ${ }^{\circ}$ 2256-5078 / Nombre abreviado: cuad.adm.

Edición Vol. $29 \mathrm{~N}^{\circ} 49$ (enero - junio de 2013)

Competitividad en empresas innovadoras de software y tecnologias informaticas/Henry Alberto Mosquera Abadía y Julio César Millán Solarte 